\title{
Narrativa
}

\section{Lo spazio ambiguo: la narrativa sarda contemporanea e la dominazione spagnola nell'isola}

\section{Roberto Lapia}

\section{CpenEdition}

\section{Journals}

Edizione digitale

URL: https://journals.openedition.org/narrativa/694

DOI: 10.4000/narrativa.694

ISSN: 2804-1224

\section{Editore}

Presses universitaires de Paris Nanterre

\section{Edizione cartacea}

Data di pubblicazione: 1 décembre 2017

Paginazione: 115-128

ISBN: 978-2-84016-289-6

ISSN: $1166-3243$

Notizia bibliografica digitale

Roberto Lapia, «Lo spazio ambiguo: la narrativa sarda contemporanea e la dominazione spagnola nell'isola», Narrativa [Online], 39 | 2017, online dal 01 décembre 2021, consultato il 14 janvier 2022. URL: http://journals.openedition.org/narrativa/694 ; DOI: https://doi.org/10.4000/narrativa.694

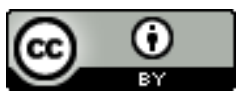

Narrativa est mise à disposition selon les termes de la Licence Creative Commons Attribution 4.0 International. 


\section{Lo spazio ambiguo: \\ la narrativa sarda contemporanea \\ e la dominazione spagnola nell'isola}

N egli ultimi trent'anni si è assistito a un profondo rinnovamento della produzione letteraria sarda. In questo contesto, è interessante rilevare un ritorno d'interesse per il romanzo a tema storico anche attraverso delle forme molto diversificate rispetto al passato ${ }^{1}$. All'interno di tale tendenza si può osservare come alcuni autori isolani abbiano deciso di focalizzare la loro attenzione sul periodo della dominazione spagnola in Sardegna, in particolare sul cosiddetto Regnum Sardiniae ${ }^{2}$, quando l'isola entrò a far parte della Corona di Spagna (1479-1720). Ora, il periodo della dominazione iberica nell'isola, fino a pochi anni fa, rimaneva ancora avvolto da una sorta di "nebbia fitta, in cui ogni tanto si apre qualche squarcio di chiarezza che ne illumina aspetti particolari"3.

In questo studio ci proponiamo di mettere al centro dell'analisi dei romanzi ambientati nel periodo della dominazione spagnola in Sardegna per tentare

1. Cfr. Marras, Margherita, “Dall'Ottocento ai nostri giorni: la parabola del romanzo a tema storico in Sardegna tra coloniale e postcoloniale", in SERRA, Patrizia (a cura di), Questioni di letteratura sarda. Un paradigma da definire, Milano, Franco Angeli, 2002, p. 205.

2. Regnum Sardiniae è il nome latino del Regno di Sardegna che fu istituito nel 1297 dal Papa Bonifacio VIII. Il regno entrò a far parte della Corona di Spagna (nata dall'unione delle Corone di Castiglia e di Aragona) nel 1479 e vi rimase fino al 1718, quando si registrò il tramonto definitivo dell'impero iberico. Rispetto a questa presa di potere della Spagna sulla Sardegna e rispetto al Regnum Sardiniae, gli storici Berlinguer e Mattone affermano che "In quell'insieme di valori, simboli, e modelli culturali che sostanziano l'identità storica della Sardegna non possono ovviamente mancare le istituzioni: innanzitutto il fatto che l'isola si sia identificata col Regnum Sardiniae, cioè con quel regno autonomo che era parte integrante della confederazione catalano-aragonese, poi della Corona di Spagna e che nel 1720 diede il titolo regale al duca di Savoia" (BERLINGUER, Luigi, MATTONE, Antonello, "L'identità storica della Sardegna contemporanea", in ID. (a cura di), La Sardegna. Storia d'Italia. Le regioni dall'Unità a oggi, Torino, Einaudi, 1998, p. XXV).

3. Maurand, Pietro, La ribellione e la rivoluzione. Sardegna spagnola e piemontese, Cagliari, CUEC, 2008, p. 11. 
di rispondere ad alcune domande: quali sono le ragioni che spingono gli scrittori sardi contemporanei a riscrivere (e rivisitare) una storia "obliata"? Quali sono le conseguenze reali e potenziali di una tale "presa di posizione"? In che modo la forma letteraria agisce sull'immaginario culturale collettivo in quanto rigeneratore di mondi, quindi di identità?

Prima di concentrarci sulle ragioni e sulle modalità del recupero della storia da parte della letteratura ci sembra necessario proporre una breve contestualizzazione storica che permetta di far luce sulle cause e sulle conseguenze della rimozione della Sardegna spagnola dalla coscienza collettiva isolana.

\section{IL CONTESTO}

Il periodo ispanico dell'isola a cui facciamo riferimento, e a cui gli autori presi in considerazione prestano attenzione, è quello compreso tra il 1479 e il 1720, ovvero l'epoca che si apre con l'arrivo al potere di Ferdinando II il Cattolico e con l'annessione del Regnum Sardiniae alla Corona di Spagna, e si chiude con il patto di Londra del 1718, un accordo che assegnò la Sardegna ai Savoia in seguito alla guerra di secessione che lacerò definitivamente il regno iberico (i Savoia presero possesso effettivo del territorio sardo solo a partire dal 1720).

Che cosa sappiamo di questa "storia" spagnola? Sappiamo che è fatta di dominazione, di colonizzazione, di discriminazioni e di subordinazioni. Nonché d'inquisizione. Ma sappiamo anche che è un'epoca di sviluppo e modernizzazione, di consolidamento sociale e, soprattutto, di complessità culturale. Tuttavia, si può asserire che questo periodo, così importante per la Sardegna, è stato in qualche modo negato e sottaciuto, caduto nell'infinito limbo di oblio e ricordo, in cui chi scrive la storia decide cosa si deve raccontare e cosa invece non è degno di essere trasmesso.

Come ha segnalato lo storico Francesco Manconi, tra il Medioevo e l'era moderna, la Sardegna è stata la regione mediterranea che visse più stabilmente e durevolmente sotto l'orbita politica e culturale della Spagna ${ }^{4}$. Questo ebbe come corollario una profonda ispanizzazione dell'isola e della società sarda in tutti i suoi aspetti ${ }^{5}$. Ciò nonostante, una pregnante leggenda anti-ispanica,

4. Manconi, Francesco, "L'ispanizzazione della Sardegna: un bilancio", in Brigaglia, Mario, Mastino, Antonello, Ortu, Gian Giacomo (a cura di), Storia della Sardegna 1. Dalle origini al Settecento, Bari, Laterza, 2006, pp. 221-237.

5. Da segnalare, per esempio, la creazione delle università di Cagliari e di Sassari tra la fine del XVI e l'inizio del XVII secolo, sotto il regno di Filippo II. Ufficialmente l'obiettivo del potere centrale spagnolo era quello di evitare agli studenti sardi lunghi 
la cosiddetta "leggenda nera", si diffuse in particolar modo in epoca risorgimentale, attraverso un'operazione ideologica degli storici sabaudi, impegnati in una lunga impresa che mirava a riaffermare l'italianità della Sardegna a discapito della sua ispanizzazione. Le idee preconcette divulgate all'epoca legavano l'ispanità all'oscurantismo, alla decadenza economica e al decadimento sociale. La Spagna diventava così il riferimento negativo, l'archetipo dei mali storici dell'isola, il capro espiatorio per eccellenza: "Si riteneva che in fondo i secoli dal Trecento al Settecento fossero secoli 'bui', di malgoverno e di decadenza, in cui non era accaduto niente che fosse degno d'attenzione da parte dello storico" .

Gli effetti di questa negazione storica e di questo particolare uso della memoria sono perdurati fino agli anni Sessanta-Settanta del Novecento, quando si assistette per la prima volta a un ritorno d'interesse da parte di alcuni storici ${ }^{8}$. In seguito, a partire dagli anni Ottanta, in un contesto in cui era in atto un forte rinnovamento, talvolta traumatizzante, in tutti gli strati della società isolana ${ }^{9}$, è stata la letteratura contemporanea sarda a manifestare il proprio interesse per

e costosi soggiorni di studio in Spagna e in Italia, e di formare in loco i futuri dirigenti destinati all'amministrazione pubblica. Cfr. VIRDIS, Maurizio, "La nascita della Sardegna quale soggetto storico e culturale nel secolo XVI", in Serra, Patrizia (a cura di), Questioni di letteratura sarda, cit., pp. 61-100.

6. Cfr. Mattone, Antonello, "Antispagnolismo e antipiemontesismo nella tradizione storiografica sarda", in Musi, Aurelio (a cura di), Alle origini di una nazione. Antispagnolismo e identità italiana, Milano, Guerini e associati, 2003, pp. 267-312, e Musi, Aurelio, "Fonti e forme dell'antispagnolismo nella cultura italiana tra Ottocento e Novecento", in Ibid., pp. 11-48. Per comprendere la genesi e gli sviluppi della cosiddetta "leyenda negra" spagnola, diffusasi in Europa e nelle Americhe a seguito del crollo dell'impero ispanico, si veda l'interessante lavoro dello storico valenciano GARCIA CARCÈL, Ricardo, La leyenda negra. Historia y opinión, Madrid, Alianza, 1992.

7. MANCONI, Francesco, "Introduzione", in ID. (a cura di), La società sarda in età spagnola 1 e 2, Aosta, Musumeci, 1992, p. 8.

8. Si vedano in particolare i lavori di Anatra Bruno, La Sardegna dall'unificazione aragonese ai Savoia, Torino, UTET, 1987, Casula, Francesco Cesare, Profilo della Sardegna in epoca catalano-aragonese, Cagliari, Edizioni della Torre, 1982, MAnConi, Francesco (a cura di), La società sarda in età spagnola 1 e 2, cit., MATTONE, Antonello, "Antispagnolismo e antipiemontesismo nella tradizione storiografica sarda", in Musi, Aurelio (a cura di), Alle origini di una nazione. Antispagnolismo e identità italiana, cit., pp. 267-312, e SorGIA, Giancarlo, La Sardegna spagnola, Sassari, Chiarella, 1982.

9. La Sardegna si dovette confrontare con un cambiamento di carattere epocale, come ha fatto notare Maria Amelia Amendola: "A partir dalla metà degli anni Ottanta la realtà sarda subisce una vera e propria mutazione antropologica [...]: i processi di modernizzazione e l'introduzione delle nuove tecnologie, lo spopolamento delle campagne, l'emigrazione, l'urbanizzazione, la crescita dell'alfabetizzazione e della scolarizzazione, la diffusione dei media e il turismo di massa" (AMEndola, Maria Amelia, L'isola che sorprende. La narrativa sarda in italiano (1974-2006), Cagliari, CUEC, 2006, p. 34). 
questo periodo, rappresentandolo e riattualizzandolo da una prospettiva che appariva discorde rispetto al passato: la storia raccontata dal basso. Ė a quest'ultima prospettiva che s'interessa questo studio, partendo dalla convinzione che la tradizione letteraria rimane uno dei luoghi principali di rappresentazione e autoriflessione, di creazione di "nuove" coscienze, finora inesistenti. Un luogo di riconfigurazione del mondo.

La data chiave della "ripresa storica" che ci interessa analizzare in ambito letterario può essere situata nel 1986, anno di pubblicazione del primo romanzo di Sergio Atzeni, intitolato Apologo del giudice bandito ${ }^{10}$. Attraverso quest'opera spartiacque, Atzeni rimette al centro della narrazione la rappresentazione della Sardegna come "mondo spagnolo", ponendo fine a una lunga diaspora della storia e dalla storia.

Sulla scia di Atzeni, altri scrittori sardi hanno intrapreso un lavoro di reinterpretazione dell'epoca iberica dell'isola, attraverso dei testi caratterizzati da una dialettica costante tra la ricerca documentaria e l'immaginazione, per dare vitalità a una memoria rimasta sin qui inerte.

Tra i maggiori protagonisti di questo recupero della memoria, meritano di essere menzionati Giulio Angioni, Paola Alcioni, Raffaele Puddu, Nicolò Migheli, Pietro Maurandi e Anna Castellino ${ }^{11}$. Le loro opere si possono inserire all'interno del filone che Giuliana Benvenuti ha definito "romanzo neostorico italiano" 12 : una narrazione che riscrive la storia da punti di vista oscurati e dimenticati, insinuandosi nelle maglie del discorso ufficiale per svelare il rimosso e i silenzi imposti. Una narrazione contro-storica, che attraverso l'indagine romanzesca rimette in discussione un'immagine immutabile del passato.

10. AtZeni, Sergio, Apologo del gindice bandito, Palermo, Sellerio, 1986.

11. Alcioni, Paola, La stirpe dei re perduti, Nuoro, Il Maestrale, 2003; Alcioni, Paola, Pala, Antonimaria, Addia, Cagliari, Condaghes, 2004; Angioni, Giulio, Millant'anni, Nuoro, Il Maestrale, 2002; ID., Le fiamme di Toledo, Palermo, Sellerio, 2006; Atzeni, Sergio, Apologo del giudice bandito, cit.; ID., "Gli anni della grande peste", in ID, Gli anni della grande peste, Palermo, Sellerio, 2003, pp. 36-47 e ID., "Una leggenda meridionale", in ID., I sogni della città bianca, Nuoro, Il Maestrale, 2005, pp. 183-190; Castellino, Anna, Mischinéddus. Storia minuscola dei chicos della ruota (1583-1600), Cagliari, AM\&D, 2006; Maurandi, Pietro, Hombres y dinero. Storie di passioni, congiure e delitti nella Sardegna spagnola, Cagliari, CUEC, 2010; Migheli, Nicolò, La storia vera di Diego Henares de Astorga, Cagliari, Arkadia, 2013; Puddu, Raffaele, Pueblo, Nuoro, Ilisso, 2004 (I ed. Cagliari, AM\&D, 2000. In questo articolo si fa riferimento all'edizione del 2004).

12. Benvenuti, Giuliana, Il romanzo neostorico italiano. Storia, memoria, narrazione, Roma, Carocci, 2012. 


\section{FINIS SILENTII}

È importante, a questo punto, precisare l'orizzonte teorico all'interno del quale intendiamo situare il romanzo neostorico sardo.

L'autorità produce discorsi, attraverso testi, immagini, opere, che provocano adesione. E su tale adesione essa riposa. Sovente questi discorsi "egemoni" escludono le vite marginali e gli oggetti di disturbo, le cui storie rimangono nel silenzio. La letteratura, con i suoi mondi immaginari o ricreati, può inserirsi nelle falle del discorso dominante per rivelare il rimosso e produrre, attraverso uno strappo che Iain Chambers definisce derridiano ${ }^{13}$, un discorso nuovo, diverso, aperto. Emergono così dei racconti che prima erano stati negati, parodiati o semplicemente ignorati. S’inizia a leggere ciò che non era ancora stato scritto ${ }^{14}$.

Ne consegue che il ruolo della letteratura è anche quello di incoraggiare una rilettura, una riconsiderazione del passato per riconfigurarlo alla luce critica del presente, ospitando quelle sfide che sembrano emergere da una complessità rimossa e che permettono di giungere a una ridefinizione e a una problematizzazione delle culture (e delle letterature stesse). La narrativa sarda contemporanea, grazie ad alcuni suoi importanti esponenti, a partire dagli anni Ottanta del Novecento, inizia a rileggere e a rivisitare la storia spagnola dell'isola, accogliendo le sfide lanciate dal lungo silenzio del racconto storico per narrare quel periodo. Attraverso questa narrativa neostorica che unisce il regno dell'affabulazione romanzesca a quello della storia, emerge tutto il rimosso con le sue complessità e potenzialità che problematizzano le costruzioni identitarie destabilizzandole e aprendole a una nuova definizione che sembra slacciarsi dall'assunto etnocentrico per spostarsi verso una evidente polifonia culturale, per "appartenere ad altro, in modo nuovo"15.

13. "Derrida ha provocato una vasta rivalutazione dei discorsi politici e culturali, particolarmente nell'area degli studi subalterni e della critica postcoloniale. Creando delle fratture nel linguaggio, contestandone la presunta unità e autorevolezza metafisica, l'opera di Derrida ha evocato spazi in cui altri mondi potrebbero apparire e incominciare a mettere insieme il vuoto che circonda l'egemone testo europeo. Sospendendo, interrogando, rinviando e differenziando il senso, Derrida ha dato la possibilità di rompere un silenzio storico e mettere in discussione la conclusione etnocentrica intenta a una verità che conduce inevitabilmente a qualche beneficio interno" (CHAMBERs, Iain, Paesaggi migratori. Cultura e identità nell'epoca postcoloniale, Roma, Meltemi, 2003, p. 43).

14. Cfr. Ibid., p. 44.

15. Cfr. Angioni, Giulio, "Appartenenze", in Contarini, Silvia, Marras, Margherita, PIAS, Giuliana (a cura di), L’identità sarda nel XXI secolo, tra globale, locale e postcoloniale, Nuoro, Il Maestrale, 2012, pp. 21-30. 
Demistificare la storia significa dunque rendere accessibile un passato negato o obliato, ma non solo. Liberare i racconti non detti vuol dire anche liberare il presente dagli stereotipi che ancora oggi pesano sulla Sardegna e sulla sua cultura, significa ribellarsi al pittoresco. Questa demitizzazione si ottiene dunque con la letteratura, che rivendica il ruolo centrale della fantasia "Perché la verità è dalla parte della fantasia, anche se smentita dalla politica reale"16.

Potremmo forse definire queste scritture come "scritture eretiche", secondo la definizione che ne ha dato Carla Benedetti nel saggio Disumane lettere: pretendere da una scrittura che essa "esprima un impensato" - un'idea di rottura - è "eresia massima" 17 . Una scrittura che mette fine alla violenza dell'oblio forzato attraverso la parola erratica, cioè una parola che non è mai data, ma si modifica nel tempo e nello spazio e dimostra l'esistenza di una "società calda", come direbbe Lévi-Strauss, perché tutte le società hanno coscienza storica, "solo che alcune lo ammettono, mentre altre preferiscono ignorarlo"18.

Una presa di coscienza della propria storia che passa per una "presa della parola"19 dell'autore, che attraverso la sua "conoscenza integrativa" 20 , fatta di finzione, storia e memoria, restituisce la voce ai morti, agli esseri senza nome (la cosiddetta "pulsione negromantica" 21 ) e permette a un'epoca dimenticata e catalogata dalla storiografia risorgimentale come "leggenda nera" di essere finalmente rivelata.

Raccontare il passato da una prospettiva differente, attraverso un punto di vista alternativo, permette agli autori sardi di sovvertire la narrazione ufficiale e di innalzare i subalterni al ruolo di soggettività parlanti all'interno del discorso storico. Ciò significa da un lato contribuire a far crescere la consapevolezza rispetto alla società sarda contemporanea ("Cosa siamo diventati oggi??, "Perché siamo diventati così?") 22 , dall'altro cogliere pienamente il suggerimento di Antonio Gramsci riguardo l'importanza, dal punto di vista civile e politico, di

16. Calvino, Italo, Una pietra sopra, in ID., Saggi. 1945-1985, vol. I, Milano, Mondadori, 1995, p. 13.

17. Benedetti, Carla, Disumane lettere. Indagini sulla cultura della nostra epoca, Bari, Laterza, 2011, p. 130.

18. LeVi-STrauss, Claude, "Histoire et ethnologie”, in Annales ESC, n. 6, 1983, p. 1218.

19. "Il s'est produit ceci d'inouii : nous nous sommes mis à parler. Il semblait que c'était la première fois" (CERTEAU, Michel de, La Prise de la parole et autres écrits politiques, Paris, Éditions du Seuil, 1994, p. 41).

20. SAID, Edward, "Introduction", in GuHA, Ranajit, SPIVAK, Gayatri Chakravorty, Selected Subaltern Studies, New York, Oxford University Press, 1988, p. 24.

21. Benvenuti, Giuliana, Il romanzo neostorico italiano, cit., p. 8.

22. Cfr. Manai, Franco, Cosa succede a Fraus? Sardegna e mondo nel racconto di Giulio Angioni, Cagliari, CUEC, 2006, pp. 204-205. 
un progressivo acquisto della coscienza della propria personalità storica da parte di strati subalterni che non sospettano neanche che la loro storia possa avere una qualsiasi importanza e che abbia un qualsiasi valore lasciarne tracce documentarie ${ }^{23}$.

I vinti, gli sconfitti della storia, non sono più topos della narrazione, ma divengono essi stessi soggetti, agenti trasgressivi. Negare la storia, o una parte di essa, significa escludere gli individui che quella storia l'hanno popolata, vissuta, e sofferta. Significa annullare delle esperienze, impedire il formarsi di una memoria condivisa, mutilare la coscienza culturale di un popolo. Così, rimettere al centro del racconto gli emarginati adesso diviene possibile grazie al controdiscorso letterario: un discorso che parte dal basso, dal frammento, dal dettaglio, per affrontare la precarietà del passato. In tal modo è possibile far affiorare le storie che sembravano prive d'interesse, instillare un dubbio sulla verità data ${ }^{24}$.

Per concludere su questa prima parte, possiamo dire che la narrativa sarda che ci racconta della Sardegna iberica costituisce una sorta di "excursus narrativo della storia spagnola" 25 che arriva al lettore come una densa costruzione poetica e intellettuale dotata di una chiara progettualità che si declina in maniera differente in ogni opera a seconda della sensibilità di ogni autore. Un excursus che rende possibile la costruzione di un quadro storico accessibile a tutti e che permette l'identificazione dei soggetti di quella storia fin qui omessi.

La letteratura si fa dunque "ginnastica della coscienza" 26 e ci permette di andare oltre le amnesie collettive e le occultazioni ufficiali del passato ${ }^{27}$.

23. Gramsci, Antonio, Quaderni del carcere, vol. IV, Torino, Einaudi, 1975, p. 1041.

24. Come sosteneva Sergio Atzeni: "Bisogna diffidare della verità, [...] soprattutto di quelli che dicono di sapere qual è la verità" (in Sulis, Gigliola, "La scrittura, la lingua e il dubbio sulla verità. Intervista a Sergio Atzeni”, in La Grotta della Vipera, Cagliari, XX, 66-67, 1994, p. 41).

25. Cfr. Porcu, Giancarlo, "Voci dalla sottostoria", in Bachis, Francesco, Pusceddu, Antonio Maria (a cura di), Cose da prendere sul serio. Le antropologie di Giulio Angioni, Nuoro, Il Maestrale, 2015, p. 243.

26. "La letteratura è ginnastica della coscienza, necessaria in un'epoca in cui l'iper-rapidità della mutazione colonizza e intorpidisce la coscienza stessa" (ZinATO, Emanuele, Letteratura come storiografia? Mappe e figure della mutazione italiana, Macerata, Quodlibet, 2015, p. 16).

27. "La Sardegna spagnola non fu una specie di buco nero, una semplice parentesi fra i giudicati e il governo sabaudo, una sorta di sospensione della storia. Ci sono tracce sicure che essa sia stata partecipe a pieno titolo del mondo iberico, delle sue particolarità, delle sue opportunità e dei suoi problemi. [...] [Oggi] s'impone una rivisitazione delle inquietudini e delle lotte della Sardegna "spagnola", affinché per essa abbia fine il regno delle favole e incominci il regno della storia" (MAurand, Pietro, La ribellione e la rivoluzione. Sardegna spagnola e piemontese, cit., pp. 10-11). 
Il filone letterario del romanzo neostorico sardo che rivisita il periodo spagnolo si presta a essere analizzato in questa prospettiva perché, pur nella sua varietà, presenta caratteri comuni. All'interno di questa produzione, una delle voci più originali è sicuramente quella di Raffaele Puddu, autore del romanzo Pueblo. Il termine pueblo è ambiguo e su quest'ambiguità l'autore sembra giocare in tutta l'opera. Difatti, "pueblo" si presta a una doppia interpretazione/traduzione, contenitore di un'ambiguità semantica di fondo: pueblo è "paese", ma pueblo è anche "popolo". Viene da chiedersi: quale "paese"? Quale "popolo"? $\mathrm{E}$ alla lettura del romanzo viene da rispondere: tutti i paesi e tutti i popoli. E viene anche da aggiungere: subalterni.

Pueblo è un romanzo storico che "non indulge a una rappresentazione stereotipa della Sardegna spagnola"28, ed è significativo che sia stato scritto da uno storico (prestato alla letteratura) ${ }^{29}$ che in questo caso decide di volgere lo sguardo oltre le versioni ufficiali della Storia.

Puddu, attraverso la sua scrittura, sembra evidenziare l'importanza dell'utilizzo e della distorsione del dato storico, spingendosi fino a quella che Bruno Anatra definiva "invenzione della storia" da parte della letteratura ${ }^{30}$ : accanto a una forte tensione alla verosimiglianza storica (non come aderenza documentaria, ma come "resa letteraria del sapore del tempo"31) si riscontra in Pueblo una particolare volontà di distorsione della realtà descritta, volta a far scattare la

28. Bazzano, Nicoletta, "La leyenda negra continua... La Sardegna viceregia nella narrativa sarda fra secondo Novecento e nuovo millennio", in Mediterranea - ricerche storiche, Palermo, anno XIII, n. 37, agosto 2016, p. 373 (si veda la nota n. 73). Bazzano, nel suo articolo, propende per una tesi opposta a quella portata avanti in questo studio. Difatti, secondo la Bazzano, "un esempio di veicolazione dell'immagine negativa della presenza spagnola è costituito dalla narrativa sarda di ambientazione storica dell'ultimo quarantennio". Gli scrittori isolani lavorerebbero, secondo Bazzano, "alla formazione del senso comune storico e no, del pubblico, non preoccupandosi, però, della verità storica del messaggio di cui sono latori” (p. 354). Insomma, Pueblo, a suo modo di vedere, rappresenterebbe una delle poche eccezioni alla regola.

29. Come afferma Ricardo Garcia Carcèl nella nota introduttiva: "Puddu ha sentito il bisogno di varcare la frontiera che separa storia e letteratura, di sottrarsi alla schiavitù di fonti documentarie che, salvo il caso di quelle processuali, sembrano ignorare gli esseri senza nome, i soggetti passivi della storia" (GARCIA CARCÈL, Ricardo, in PudDu, Raffaele, Pueblo, cit., p. 6).

30. Cfr. Anatra, Bruno, "L’invenzione della storia", in Marci, Giuseppe, Sulis, Gigliola (a cura di), Trovare racconti mai narrati, dirli con gioia, Cagliari, CUEC, 2001, pp. 81-86.

31. Ibid. 
cosiddetta "scintilla creativa". Si veda ad esempio l'uso di toponimi e antroponimi immaginari:

Calcolò che, rallentati dagli agnelli, gli uomini del marchese avrebbero impiegato un giorno per raggiungere l'altro versante de Su Monte, e li immaginò arrancare per la ripida erta di Mollosu. Sarebbero passati sotto le rocce di Zinnigas, e là sarebbe andato ad aspettarli ${ }^{32}$.

Vi è uno "scarto" (che in questo passaggio è rappresentato dall'utilizzo dei toponimi immaginari: Su Monte, Mollosu, Zinnigas), il quale rappresenta appunto l'intervento dello scrittore che tratta e manipola la materia documentaria in modo personale e creativo.

Puddu procede in Pueblo a inserire la Sardegna nel complesso reticolo territoriale, sociale e culturale dell'impero spagnolo. Un impero multietnico e multiculturale a cui i sardi presero parte attiva. L'intento dell'autore appare quello di far risaltare non tanto (o non solo) la complessità di quel "mondo spagnolo" di cui l'isola era partecipe, quanto l'idea di una cultura sarda in continuo contatto, conflitto e intercambio attivo con culture altre, seppur nella sua veste "periferica" marcata da un'arretratezza di fondo; come se la Sardegna fosse una periferia nel cuore della storia.

Al centro del racconto troviamo la figura del giovane pastore Diego Flores di Cadennaghe (paese immaginario del centro della Sardegna) che, insofferente per la sua condizione di servo feudale, e in seguito a un omicidio commesso per vendicare la morte del padre, si arruola nel Tercio di Sardegna ${ }^{33}$ e s'imbarca da Caller (Cagliari) insieme ai tanto temuti fanti spagnoli che si battono per il re Filippo II e per la chiesa di Roma.

Il percorso di Diego Flores è quello di un subalterno che si ritrova al centro delle vicende del suo tempo, accompagnato dal ricordo ostinato della sua terra e da uno sguardo trasognato della storia. Come soldato di sua Maestà si troverà a combattere in Spagna, quindi in mezzo all'Europa eretica, e finirà i suoi giorni (e i suoi sogni di ritorno in patria) nelle sterminate lande americane, dove all'epoca si sviluppavano le nuove mire espansionistiche della Corona. Si assiste in tal modo a un continuo movimento di dilatazione spaziale che mette al centro del narrato un'ibridazione geografica di lingue, di luoghi, di contaminazioni ricorrenti.

Pueblo, pur essendo un romanzo storico, è un romanzo breve, caratterizzato anche da una forte mescidanza linguistica, con una compenetrazione continua

32. Puddu, Raffaele, Pueblo, cit., p. 17.

33. Il Tercio di Sardegna era un'unità militare spagnola sita nell'isola. 
tra il sardo, lo spagnolo e l'italiano. La mescidanza è più intensa nella prima parte del testo, mentre nella seconda parte risulta meno manifesta. Benché l'italiano rimanga comunque l'idioma maggioritario e predominante, ci troviamo in assenza di una manifesta soluzione di continuità tra le differenti parlate; effetto che l'autore ottiene, in parte, grazie alla scelta dell'uniformità tipografica, che a volte rende poco agevole la lettura.

Insomma, l'operazione letteraria di Puddu si fonda sullinvenzione della lingua, cui si unisce l'invenzione della storia ovvero la deformazione dei dati documentari e del materiale storico: ne consegue una manipolazione costante, dei materiali linguistici e dei fatti, un complesso di deformazioni che finisce per dar vita a una pagina di storia dotata di coerenza e tangibilità, che esiste grazie alle manipolazioni che competono alla letteratura. Questo tipo di narrazione, densa, mescidata, alterata, fornisce numerosi spunti di riflessione, tre dei quali sono al centro della nostra indagine: la vicinanza tra i sardi e gli altri subalterni (in particolare gli indios delle Americhe); la problematizzazione della relazione Sardegna/Italia; infine, una nuova idea di "spazio isolano".

Iniziamo dal primo punto: in Pueblo il legame tra i sardi e gli altri popoli sottomessi, pur nella loro diversità, appare naturale, come già in altre opere della produzione neostorica presa in considerazione, come ad esempio Millant'anni di Giulio Angioni. In Pueblo il vincolo sembra stringersi prima con gli abitanti dell'Estremadura ${ }^{34}$, quindi con gli indios:

Quella città somigliava a Caller, invece si chiamava Veracruz. [...] Diego fece caso all'aspetto strano di tutta quella gente curva sotto pesi da troncare la schiena o seduta per terra a gambe incrociate. Gli occhi sembravano ferite d'arresoja sul punto di rischiudersi, la loro pelle era più scura ancora di quella dei naraboliesos, figli dei moros che non avevano avuto voglia di farsi la salita fino a Cadennaghe e se n'erano restati lì in pianura a divertirsi con le donne di lì. Solo, invece di avere i capelli ricciuti, ce li avevano lisci e lunghi, ed era difficile distinguere i maschi dalle femmine $e^{35}$.

Il cortile era pieno di quelli che, in cuor suo, Diego continuava a chiamare naraboliesos, e che per gli spagnoli erano indios, [...] le loro facce erano tristi comente s'annada mara ${ }^{36}$.

34. "Giunsero i rincalzi e furono distribuiti tra le diverse compagnie. La maggioranza degli uomini che toccarono a Lopez de Montoya erano bisoños di un paese che si chiamava Estremadura ma avrebbe potuto essere Cerdeña: il loro aspetto selvatico parlava di greggi, di pascoli e di solitudine" (Puddu, Raffaele, Pueblo, cit., p. 55).

35. Ibid., p. 81

36. Ibid. 
Quando, nel deserto americano, un terremoto distrugge il Tercio, Diego Flores sopravvive e finisce nel pueblo indio, accolto senza resistenze dai capi del villaggio. Diego si ritrova a cantare in sardo davanti a tutti gli abitanti e viene accettato come fratello. Un mutuo riconoscimento che è altresì "muto" riconoscimento, vista la discrepanza linguistica tra Diego e gli indios. Non si parlano perché non hanno una lingua in comune, eppure si capiscono. Egli si rende conto che anche gli indios sono uomini, che sanno coltivare la terra e sanno cacciare; mentre per gli spagnoli non erano altro che bestie, animali selvatici da ammaestrare e convertire alla fede di Dio Nostro Signore. E agli abitanti del pueblo Diego Flores darà dei nomi di chiara matrice sarda (o latina) non sapendo nominarli in altro modo:

Ecco come Diego era entrato nella famiglia di re Felippu. Sì, perché Mundeddu e la ragazza della collana, che, siccome il nome non lo sapeva dire chiamava Maria, erano tutt'e due figli suoi, e ne aveva anche altri, grandi e piccoli, fatti con mogli diverse ${ }^{37}$.

Ed è proprio nel tentativo di salvare il re indio "Felippu", messo al rogo dagli spagnoli, che Diego verrà ucciso dal fante Machuca dell'Estremadura, in una specie di "cortocircuito subalterno" finale. Questo passaggio fotografa, inoltre (in maniera non retorica), il differente modo di ragionare dell'occupante e dell'occupato: una visione del mondo opposta, una mentalità estremamente distante.

Di notevole interesse è anche la relazione che Puddu disegna all'interno del testo tra i sardi e gli italiani, quindi tra la Sardegna e l'Italia. Durante un periodo di stanza nelle Fiandre, Diego Flores ucciderà un italiano a causa del suo atteggiamento violento e machista nei confronti di una donna del luogo.

Italianos, - bofonchiò Ortega con un'alzata di spalle [...].

Diego parò tre stoccate, fece una finta al volto e la sua spada affondò quasi che non solo il giubbotto, ma tutto l'uomo, fossero fatti di velluto. Mentre s'afflosciava al suolo, gli occhi, da feroci che erano, si sgranarono di stupore [...].

Ortega ruppe il silenzio: a quest'ora, l'italiano starà raccontandole a Belzebù, le sue fanfaronate ${ }^{38}$.

37. Ibid., p. 96.

38. Ibid., pp. 47-48. 
L'omicidio è anche la conseguenza della totale assenza di empatia tra Diego e il gruppo di italici ${ }^{39}$ presenti in una vecchia bettola fiamminga, che Diego accuserà di avere un atteggiamento da "padroni”" alla stregua degli spagnoli. Egli li considerava estranei, e il loro modo di fare, il loro strillare, gli "facevano venire il sangue alla testa" ${ }^{40}$. Quando gli amici dell'ucciso provano a scagliarglisi addosso, sono gli altri militi spagnoli a difenderlo, ed è un gesto che rientra nella logica storica dei fatti poiché gli spagnoli e i sardi appartengono allo stesso Impero.

Questo episodio ha un portato simbolico forte perché il legame (spesso traumatico) che sembra unire la piccola "provincia" sarda al Regno spagnolo conduce a una possibile lettura allegorica di fondo. In effetti, il rapporto tra la Sardegna e l'Italia viene rimesso in discussione e il narratore lascia spazio all'interpretazione del lettore senza prendere posizione, ma invitandolo implicitamente a farsi una domanda scomoda, nel segno dell'ucronia: e se la Sardegna fosse rimasta spagnola? O meglio: e se la Sardegna non fosse mai diventata italiana? ${ }^{41}$

Dal racconto di Puddu emerge anche che gli strati popolari della società sarda accettavano sistematicamente la presenza spagnola nell'isola senza porsi troppe domande al riguardo, come se l'autore volesse da un lato smentire l'idea etnocentrica di una cultura antropologicamente impenetrabile e residuale nonostante gli incontri con altri popoli e le tante dominazioni subite, una cultura esclusa dai processi della storia anche grazie a (o a causa di) una "costante resistenziale" millenaria dei sardi contro ogni invasore ${ }^{42}$; dall'altro lato è come se volesse rimettere in questione il rapporto con l'Italia, in particolare il discorso "risorgimentale" italianizzante teso a cancellare e a negare ogni tipo di influenza "straniera" anteriore all'arrivo dei Savoia nell'isola.

Infine, l'ultimo spunto di riflessione: quello spaziale. Diego Flores lascia la Sardegna e va a combattere prima in giro per l'Europa "spagnola" e in seguito nelle Americhe oggetto di colonizzazione. Il suo sarà un viaggio senza ritorno perché in Messico troverà la sua fine. Oltre al paradosso di una morte in territorio colonizzato in cui arriva da colonizzatore, senza tuttavia esserlo davvero, l'impossibile ritorno nella sua terra può essere letto come un'ulteriore allegoria della condizione storica della Sardegna, che una volta trovatasi all'interno di

39. Si tenga presente che il romanzo è ambientato nel XVI secolo, all'epoca non esisteva ancora un'entità statuale e nazionale italiana.

40. Puddu, Raffaele, Pueblo, cit., p. 47.

41. A tali questionamenti si potrebbe aggiungere un dubbio altrettanto incomodante, sotto il segno della distopia: e se la Sardegna non fosse mai diventata sarda?

42. Come sosteneva lo storico Giovanni Lilliu. Si veda a proposito: LiLliu, Giovanni, La costante resistenziale sarda, Nuoro, Ilisso, 2002; I edizione: Cagliari, Fossataro, 1971. 
un impero, in posizione subordinata, si troverà coinvolta in un cambiamento da cui non si torna indietro: non più "isola-mondo" (sufficiente a se stessa) ma isola-nel-mondo, con relative conseguenze a livello culturale, sociale, linguistico, quindi identitario; con uno "spostamento di frontiera" che le fa perdere la sua storica insularità o "isolitudine"43. La Sardegna, diremmo con Chambers,

si trasforma ineluttabilmente da monumento che celebra se stesso a intersezione, momento d'incontro, luogo di passaggio di una rete più ampia. Mollati gli ormeggi l'isola comincia ad andare alla deriva, entra in altri racconti ${ }^{44}$.

\section{Conclusione}

Si configura così l'immagine di un "luogo di storie" che permettono di giungere altrove. Una riflessione come questa, attenta alla dimensione spaziale, consente di aprire una nuova prospettiva "geografica", in cui ci potremo muovere in ricerche successive. Partendo dal presupposto che, come sostiene Francesco Fiorentino, "ogni spazio è sempre anche uno spazio simbolico prodotto dai testi che l'hanno descritto e investito di significati" 45 , sarebbe interessante tentare di comprendere in che modo la rappresentazione dell'epoca spagnola della Sardegna nei romanzi sardi modifica l'idea dello "spazio isolano" nell'immaginario collettivo sardo.

Per il momento possiamo affermare che, all'interno di un più generale rinnovamento della narrativa sarda contemporanea, le scrittrici e gli scrittori che decidono di concentrarsi sul periodo spagnolo dell'isola rigenerano, attraverso la contro-narrazione, un passato taciuto, partendo dalle (molte) rovine rimaste e ridotte al silenzio. Riteniamo perciò, con Franco Fortini, che "anche i morti, stupefatti, [sono stati] scossi nei loro nascondigli. La storia presto sarà tutta scritta di nuovo" 46 , presupponendo che tale ruolo, di riscrittura e di identificazione

43. Concetto liberamente ispirato al titolo di un libro di Laura Fortini e Paola Pittalis. Cfr. Fortini, Laura, PitTalis, Paola, Isolitudine. Scrittrici e scrittori della Sardegna, Guidonia (RM), Iacobelli, 2010.

44. Chambers, Iain, Paesaggi migratori, cit., p. 123. In realtà Chambers in questa citazione si riferisce a Napoli, ma l'idea di un'isola che entra in altri racconti ben si adatta anche alla realtà sarda.

45. Fiorentino, Francesco, Solivetti, Carla, "Premessa", in ID. (a cura di), Letteratura e geografia. Atlanti, modelli, letture, Macerata, Quodlibet, 2012, p. 9.

46. Fortini, Franco, Extrema ratio. Note per un buon uso delle rovine, Milano, Garzanti, 1990 , p. 83. 
dei soggetti di quella storia (quindi dei protagonisti stessi delle vicende dell'epoca), spetti alla letteratura poiché essa è capace di accedere alle sfere dell'esistenza e soprattutto dell'esperienza, a differenza della storiografia. Perché la letteratura è svincolata da costrizione, è relativamente libera, abitata da più voci e da più punti di vista, ricca di contraddizioni interne e di aporie, luogo dell'alterità.

Diciamo allora che la narrativa sarda neostorica odierna tenta, attraverso un complicato esercizio dialettico tra fantasia e realtà storica, di trovare il filo conduttore che renda coerente questa storia "spagnola" dell'isola; o meglio, queste storie. Ciò dovrebbe permettere di superare da un lato il rimosso (o una parte di esso), dall'altro il dato bruto, ovvero quell'ammasso di notizie, dettagli e tracce raccolti ma ancora privi di voce e di coesione.

Come abbiamo sottolineato, la rivisitazione letteraria dell'epoca spagnola della Sardegna si declina in maniera diversa in ognuna delle opere, a seconda della sensibilità e dello stile propri di ogni autore. Per contro, si può affermare che l'insieme di queste narrazioni (che hanno una certa coerenza e organicità complessiva) permette di sottrarre l'isola all'insularità per aprirla al mondo; ciò fa sì che certe concezioni identitarie oppressive perdano finalmente la loro condizione sisifica di eterna chiusura, di un’identità come diversità nel senso di esclusione dal mondo, dalla storia.

Ci troviamo così di fronte a uno spazio (l'isola) che ormai è divenuto ambiguo: ovvero "uno spazio in cui le differenze hanno udienza, in cui gli oratori e le sintassi della conversazione corrono il rischio di essere modificati" ${ }^{47}$. In cui le identità non sono mai date, ma vengono rimodellate di continuo, sotto il segno dell'ibridazione e della stratificazione del patrimonio culturale condiviso.

Roberto LAPIA

Université Paris Nanterre (CRIX - EA 369 Études Romanes)

47. Chambers, Iain, Paesaggi migratori, cit., p. 45. 